\title{
SIMULATION AS SUPPORT FOR PRODUCTION PLANNING IN SMALL AND MEDIUM ENTERPRISE: A CASE STUDY
}

\author{
Roberto Mosca \\ Lucia Cassettari \\ DIPTEM Dipartimento di Ingegneria della Produzione, \\ termoenergetica e Modelli Matematici \\ Via All'Opera Pia, 15 \\ University of Genoa \\ Genoa, GE 16145, ITALY
}

\author{
Roberto Revetria \\ Gianluca Magro \\ Centro di Eccellenza per la Logistica Integrata \\ Via Balbi, 22/7 \\ CIELI \\ Genoa, GE 16100, ITALY
}

\begin{abstract}
The proposed application is related to an Italian small factory that produces, assembles, and sells mechanical components for awnings. In such factories BPR (Business Process Engineering) usually takes place without the support of Modeling \& Simulation although such methodologies have proved to be very effective and helpful. Main reasons for that have to be investigated in the high costs usually associated with a simulation study, especially for data collection, model building and model validation. In order to avoid this problem a general-purpose simulation framework was designed enabling self-build according to production process information stored in a relational database. Moreover, the simulation model was used in conjunction with a statistical analysis tool in order to build the relationship among selected parameters and the proposed objective function by mean of Response Surface Methodology and $2^{\text {nd }}$ order regressions meta-models. Authors applied the proposed schema to several industrial applications obtaining interesting results.
\end{abstract}

\section{INTRODUCTION}

This paper presents a case study related to a project for the optimization of the manufacturing process in a small company working in the area of manufacturing producing mechanical components and finished products for awnings. Beside a simple production process (drilling, machining and assembly) the company is facing several problems related to poor work organization and lack of a systematic production planning and control. The authors successfully applied Modeling and Simulation in order to build Decision Support Systems able to quantify the effect of the management on the entire production process and its economical aspects (Baqnks et al. 2001) In the proposed approach well-known best practices designed for bigger industries have been spe- cially tailored to fit the S\&ME requirements in term of costs and implementation time. Small size of the company is a quite common aspect in the Italian industry where familyruled companies are often acting as outsourcers in the manufacturing. With only 7 workers direct and 8 machining centers this implementation can be regarded as an interesting base case for many real life applications. The proposed simulation approach was implemented in Arena 8.0 according to 3 main phases.

The first of these related to data collection by identifying work flows and process times of the various operations while identifying the more appropriate statistical distribution. Then for each data set we have to find a statistical distribution. Second and subsequent phase involves the implementation of the model as well as the integration of the simulator with ad hoc relational databases designed for supporting verification and validation procedures. The simulation model is, in fact, connected to the database in order to overcome the limits of the generally available simulation package in reporting and result collection. Starting from a simulated production planning a production order is generated, looped through a two-part family bill (divided into 4 sub levels) until it generates an order for every sub product giving it a priority attribute. Then the order is split into several suborders representing each entity to be produced. When an entity is produced it can free the top level entity which proceeds in the system, be matched with another entity (representation for the assembling) and together free the top level entity or exit the system updating the data base. This system was able to elaborate about 320 kinds of elements for a planning horizon of three years (almost 20,000 orders/year planned and 550,000 elements produced). The model logic divided in 52 sequences for a total amount of 1284 SIMAN objects was implemented in a generalized way in order to foster flexibility; the Arena building blocks, in fact, have been used to simulate an high level flow shop where an tailored entity is driven directly by an attributes set 
defined in a relational database; such an approach can be adopted to a wide variety of industrial application without a massive simulation effort.

In the third part of the project the functional relationship between various factors have been investigated in order to find better configuration for the manufacturing process as well as investigate the better planning process. Such phase was implemented via response Surface Methodology by using a quadratic model for a central composite design of six factors and two center points for a total of 46 runs.

\section{THE INDUSTRIAL CASE STUDY}

For the proposed case study the name of the factory was restricted for non disclosure agreement and indicated here as "FACTORY". The emerging of new competitors, and the strength of euro caused many problems in the mechanical area above all in the north-east of Italy. Investment in innovation represents today like a necessity for the business survival; however small industries have insufficient financial resources and so innovation can be very difficult.

The FACTORY competes in this political and geographical context, and needs in some way to improve itself to survive. FACTORY is a noted brand in the sector of awnings but today its qualitative standard and productive activities are poor compared with its competitors. The production process is straightforward, based on elementary and repetitive assembly operations, and so the labour is not specialized. There is standardization neither for the processes nor the flows; there isn't any optimization in the lot size; moreover, every operation is generally done in several alternative ways involving different times. No physical warehouse is controlled by any EDI procedure; there is neither references codification nor automated inventory control.

There is a lack of documentation in the material bills while the planning and control activities are based mostly on the experiences and the skills of the worker's chief; so the production planning effectiveness is minimal. For these reasons lead time far from its minimum and, consequently, the improving margins are considerable. Several questions arise from the present situation:

- New management is ready to invest but where?

- What investment is the better?

- How much return of investment is expected in the next ten years buying a new machining centre?

- It is necessary to pay for extra shifts?

Primary goal of the implemented simulation model are: to conduct a full scale What If analysis, to increase the company knowledge on the system, to identify the key parameter and to evaluate perspective returns of investments.
This is because the project has to be considered in a more vast view outside the basic study of queues, flows and machining cycles typical to the classical approach, in this way the model needs to be, at the same time, strong and flexible. These targets must be obtained with simple schemes and with the help of the clearness of animations (figure 1) in order to enhance the comprehension that fosters the model validation.

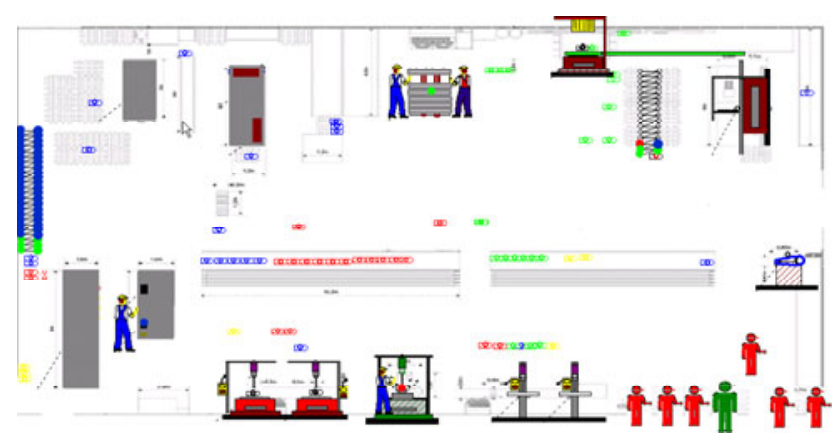

Figure 1: Part of the Simulator GUI

\section{BUILD THE SIMULATION MODEL}

The author activity has been in a first time a data collection and a study of the process beside the manufacturing plant for three months quite intensive as hour/man in the orders of $10 / 12$ for day in this phase in addition to have understand with great detail the processes and the problems (about a month) primary data has been collected concerning the flows, the processes and the times of each single operation seen. Data collection was carefully conducted in order to not interfere with the production reaching a good level of fidelity.

The average number of measure for each operation was 30 , reaching a total data collection on more than 117,600 seconds investigated. This phase is very important since it allows a full comprehension of the simulated system enabling the simulationist to better understand the underlying processes and avoiding the risk that the modeler follows his vision of the system. It's well appropriate as feedback with the results of the plant but also with people that have more experience of the processes.

A full scale distribution fitting process was adopted in order to identify the best distribution for the processing time, and a relational database was implemented for hosting the production planning. This phase was necessary as starting point for the upcoming production planning control that is under implementation.

Generally speaking timing distribution were assumed from triangular model where no other information except expert opinion was present.

This data have been collected following ad hoc schemas where sequences, resources seized and the mean time for each operation were kept. In other sheets are detailed 
the data collection results, a picture of every phase (figure 2 ) and the graph of the production flows (figure 3 ):

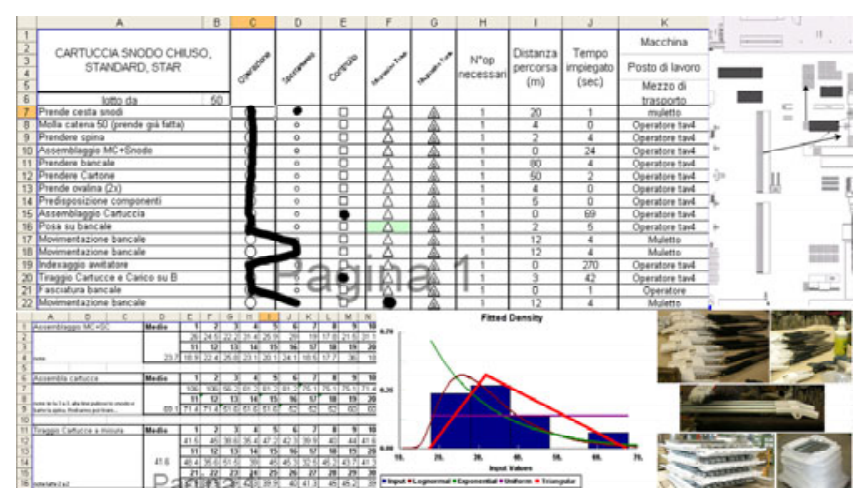

Figure 2: Data Collection Schemas

The production's process is administrated with a relational data base (MS Access 2003) in order to facilitate data entry and consistence checks.

The first table "Giorni" contains all the admitted dates from $01 / 06 / 2005$ to $31 / 05 / 2012$ (2557 days divided into 7 years. The user then can set the exceptions using Arena's scheduler. This choice enables the users to add and remove dates easily according to their needs. Another table collects the production planning as coming out from the future available production planner. A planned order is available as records structured by IDOrder, Due Date, IDProduct, Requested Quantity and Priority. Another table structure encompasses the multi-product, multi-level bill of material. Realized in two physical levels (top and bottom): logically the same product can be both in the top and bottom level to realize, practically, a multi-level family bill. The family bills include 523 record sets.

Another very important function of the relational database is to serve as virtual inventory from which are subtracted delivered products and to which are added produced elements (assembled parts or semi-finished parts) for each of the 343 products kinds entering the system (ranging from the finite products to the half-worked and the raw materials). Another table works as a join between the data base and the model. This table contains the SIMAN attributes for each entity: the sequence followed and the index related to the distribution of each process done. In fact SIMAN needs to use numbers instead of String attributes and the conversion is done inside the database.

The last table is used to collect results; as we can see in the model all the entities are focused on the same final block where the database is updated collecting for each entity the lead time and the produced quantity. At the end of each simulation run such table will contains 15,958 records reaching during simulation run a maximum of 88,865 records (including half-processed parts).

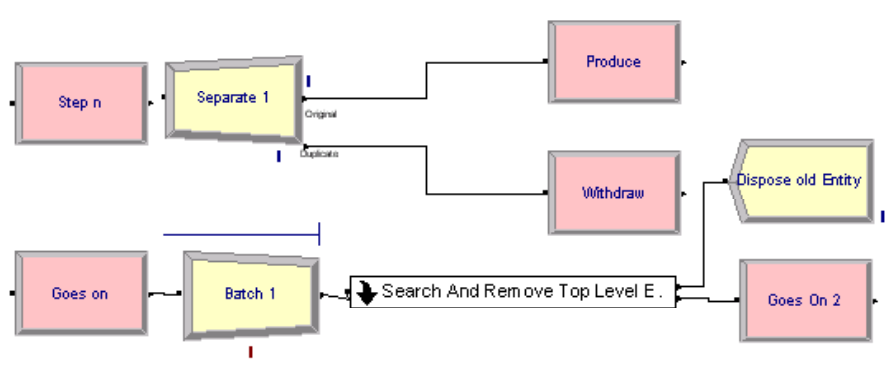

Figure 3: An Excerpt from a Sub Simulation Window

The model is structured starting from the production order generation using four nested cycles in a separate VBA module connected to SIMAN. When the order is generated two attributes (according to the inventory level and the priority) are associated with the entity: the quantity to produce and the quantity to keep. Products with higher priority (and higher added value) are final assembled and shipped as soon as possible while low added-value products (generally semi-finished parts) are machined when resources become available (Mosca, Revetria and Tonelli 2000). This one-piece flow is enhanced by an internal MRP based algorithm that schedules sub assembly and parts production according to their final due date. After the order generates the different entities with their attributes (entities that represents the different components to produce in order to satisfy the planned order), they are sent part to a logical warehouse and part to the first module of their sequence. The sequences edited are 52 and the route to take is given to the entity as an attribute. The model is structured as a flow shop where all the routing, timing and sequences information are given from the database. Each entity follows its sequence, seizing and releasing workers moving between two modules and seizing and releasing the right resource in the process module for the time established by its SIMAN attribute and for a delay dependent on the quantity to produce.

When an entity need to be produced it is split into two entities: the first one tries to withdraw the product from the inventory, the second one follows its production sequence until this sequence ends; then the entities batch together and return a unique object in the model. Now the entity is guided to two choices according to its priority: it can update the warehouse (or the table of the finished products) or can be used to produce the father component. In this second case it eventually matches with another entity (in order to represent the assembly), searches in the logical warehouse and frees his parent entity according to the OrderID. Each machining centre is modeled using preemption rules and/or with different seizing rules according to the needs of the production process. 


\section{IDENTIFYING RELATIONSHIPS AMONG PRODUCTION FACTORS}

In a third part of the project the functional relationships between various factors have been investigated in order to find better configuration for the manufacturing process as well as investigate the improved planning process. The table of the results is elaborated by an Excel program in Visual Basic for Application to obtain the target function. For each one of the 15,958 jobs produced an objective function presented was calculated in order to identify critical factors and to investigate potential relationships among production factors.

Before starting the experimental campaign a complete VV\&A procedure has been established. Logic check and software debugging were extensively used; expert judgment, walkthrough and Turing Tests served as basic Validation methodologies.

Mean Squared Pure Error time evolution was adopted in order to identify optimal duration for the simulation runs, experimental results shown typical "knee" behavior identifying the correct estimation (figure 4).

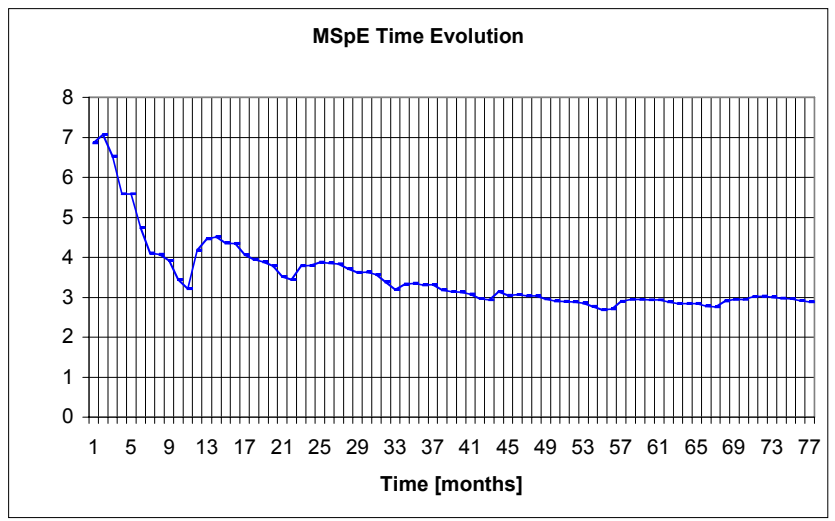

Figure 4: MSpE Curve for the Implemented Model

Investigated factors have been considered the total number of assembly centers (2-4), the work capacity of a grinding machine (2-4), total number of human resources (7-9) and the overall production volume $(0.80-1.2)$.

As can be seen, investigated factors range from work organization to resource management up to production planning scenario. Implemented objective function involves the evaluation of the produced value and the WIP obtained weighted by the lot priority, the line used and product type. Penalty factors takes into consideration human resource hired, total number of assembly station available and total number of grinding machines. Other penalty apply for improper respect of the targets calculated by the ratio among the value of the producible products on the achieved production value. Last coefficient introduces penalty for delayed productions in order to take into consideration the proper scheduling performances.
In the Objective Function (1) the following coefficients were considered:

- M: simulation length in months (in the proposed case 96);

- L: number of production lines

- $P_{\mathrm{j}}$ : items produced on line $\mathrm{j}$-th

- $\Pi_{\mathrm{kji}}:$ priority of product batch $\mathrm{k}$-th, on line $\mathrm{j}$-th over month i-th;

- $\mathrm{me}_{\mathrm{k}}$ :importance (weight) of item k-th;

- $\mathrm{ml}_{\mathrm{j}}$ : importance (weight) of line $\mathrm{j}$-th ;

- $\pi_{\mathrm{h}}$ : priority class;

- $\mathrm{Q}_{\mathrm{kji}}:$ batch size of product batch k-th, on line j-th over month i-th;

- $\lambda$ : general inventory cost;

- $\mathrm{I}_{\mathrm{kji}}$ : average inventory for product $\mathrm{k}$-th, on line $\mathrm{j}$-th over month i-th;

- $\theta$ : importance (weight) for total number of human resource hired;

- $\mathrm{N}_{\mathrm{i}}$ : total number of human resource hired on month i-th;

- $\xi$ : importance (weight) for total number of assembly work centers available;

- $\mathrm{F}_{\mathrm{i}}$ : total number of assembly work centers available on month i-th;

- $\zeta$ : importance (weight) for total number of grind station available;

- $\mathrm{A}_{\mathrm{i}}$ : total number of grind station available on month i-th

- $\chi:$ importance (weight) for total achieved schedule;

- $\mathrm{S}:$ Scheduled production;

- $\mathrm{R}$ : Realized (achieved) production;

- $\varphi$ : importance (weight) for delays in delivery;

- $\mathrm{T}_{\mathrm{jik}}$ : delay between product $\mathrm{k}$-th, line $\mathrm{j}$-th and month i-th delivery and due date;

- $\tau_{\text {jik }}$ : maximum acceptable delay for product $\mathrm{k}$-th, line $\mathrm{j}$-th and month $\mathrm{i}$-th delivery and due date;

$$
\begin{aligned}
& y=\sum_{i=1}^{M}\left\{\sum_{j=1}^{L} \sum_{k=1}^{P_{j}} \sum_{h=1}^{\Pi_{k j i}}\left[m e_{k} \cdot m l_{j} \cdot \frac{1}{\pi_{h}}\left(Q_{k j i}-\lambda I_{k j i}\right)\right]\right\}+ \\
& -\theta N_{i}-\xi F_{i}-\zeta A_{i}+ \\
& -\chi \frac{\left\{\sum_{j=1}^{L} \sum_{k=1}^{P_{j}} \sum_{h=1}^{\Pi_{k j i}}\left[m e_{k} \cdot m l_{j} \cdot \frac{1}{\pi_{h}}\left(Q_{k j i}-\lambda I_{k j i}\right)\right]\right\}_{S}}{\left\{\sum_{j=1}^{L} \sum_{k=1}^{P_{j}} \sum_{h=1}^{\Pi_{k j}}\left[m e_{k} \cdot m l_{j} \cdot \frac{1}{\pi_{h}}\left(Q_{k j i}-\lambda I_{k j i}\right)\right]\right\}_{R}}+ \\
& -\varphi \cdot \min \left(T_{k i j}, \tau_{k i j}\right)
\end{aligned}
$$

This objective function was implemented in a post processing module and investigated using Design of Ex- 
periment and Response Surface Methodology (Steppan et al. 1998). Chosen quadratic model was obtained from a central composite design of four factors and six center points for a total of 30 runs using the Essential Regression $^{\mathrm{TM}}$ add-on for MS-Excel and with the help of StatEase $^{\mathrm{TM}}$.

Response Surface Methodology applied to the proposed exercise outlined the significance of two factors: the planning multiplier and the human resources hired. The regressive meta-model is presented in figure $5, \mathrm{~F}$ test has a significance of $1.41268 \mathrm{E}-05$ : definitely less than the cutoff value of 0.05 . In such way is possible to conclude that the relationship found by the meta-model is significant. On the other hand, however, the significance for the Lack-OfFit test $(\mathrm{F}$ value $0,000933<0.05)$ clearly tells that a better model should be identified.

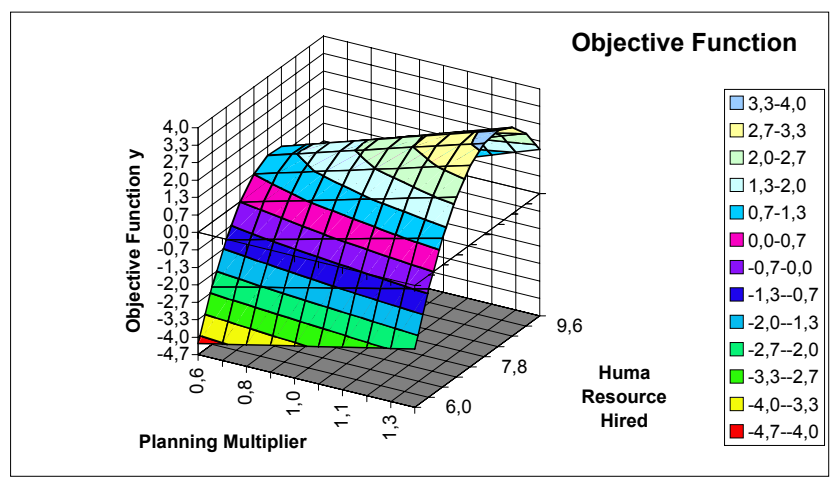

Figure 5: Regressive Response Surface for the Implemented Model

For this reason both linear and cubic models were tested without significant improvement in the performances. At this point it is clear that polynomial models needs to be substituted by more flexible meta-models and an Artificial Neural Network was adopted (Bruzzone, Giribone and Revetria 1999).

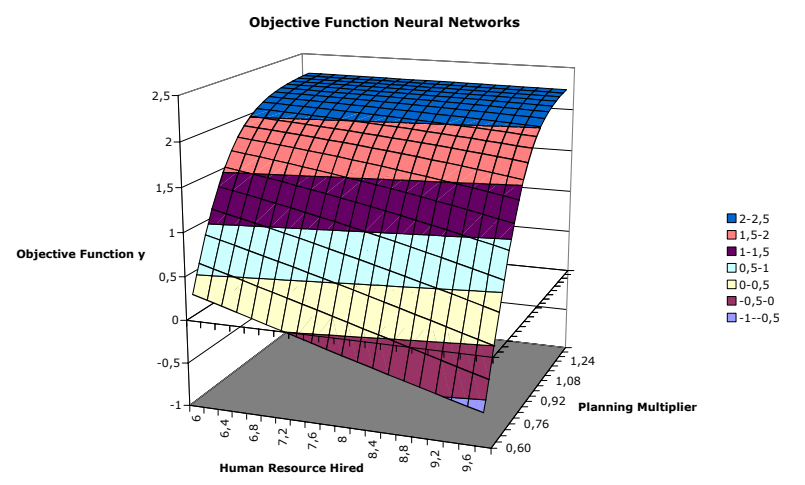

Figure 6: Artificial Neural Networks Meta-Model

Artificial Neural network meta-model was built starting from a Single Hidden Layer Full Connected Feed
Forward network with four PE on the input layer, two PE on the hidden layer (Sigmoid Activation Function) and one $\mathrm{PE}$ on the output layer (Linear Activation Function). Training set was randomly chosen as $92 \%$ of the total data available while $8 \%$ was kept as test set. Results showing a low error rate at the end of the 500,000 cycle training and a good generalization skill. Particular test error was kept around $11 \%$.

As is possible to see by comparing the two metamodels (that appears rotated $90^{\circ}$ to each other) some similar behaviors have been identified by the two techniques showing improvement possibility in the overall performances of the production process. Performances of the Neural Meta-Model may be increased moving to a $4 \mathrm{k}$ factorial design rather than by using CCD.

\section{CONCLUSIONS}

The paper demonstrates practically the applicability of the proposed methodology to a real-life application. In particular the implemented simulation framework demonstrates an high degree of flexibility serving different simulation exercise with minor changes. The workflow structured in a relational database instead of into the simulator itself fosters reusability and interoperability reducing dramatically the time and cost requirement of a complex simulation application.

Obtained results have shown great potentiality in identifying relationship among KPI and ad hoc objective function enabling managers to easily identify and evaluate strategies and criticalities.

Comparisons between Artificial Neural Networks and traditional Full Quadratic Meta-Models have been presented showing congruent results and offering alternative views of the real Response Surface.

A real life application was presented and discussed serving as base case for further implementation of the proposed methodology.

\section{ACKNOWLEDGMENTS}

The authors acknowledge additional contributions by Guido Guizzi from Università di Napoli Federico II, Luca Marazzi from CIELI and Grazia Torre for her support and patience along experimental campaign.

\section{REFERENCES}

Banks J., Carson J., Nelson B., and Nicol, D. 2001. Discrete-event system simulation, third edition, Prentice Hall

Bruzzone A.G., Giribone P, Revetria R. 1999. Design and management issues in the development of new workshops using web-based simulation and neural net- 
works Proceedings of FAIM99, Tilburg (NL) June 2325

Bruzzone A.G., Mosca R., Orsoni A., Revetria R. 2001. Forecasts modelling in industrial applications based on AI techniques, International Journal of Computing Anticipatory Systems (extended from Proeedings of CASYS2001, Liege Belgium August 13-18), Vol 11, pp. 245-258, ISSN1373-5411

Mosca R., Revetria R., Tonelli F. 2000. Simulation for resource allocation Proceedings of the Business and Industry Simulation Symposium ISBN 1-56555-199-0 Washington D.C., April

Steppan D., Werner J. and Yeater R. 1998. Essential regression and experimental design for chemists and engineers Available via <http: / /www. jowerner. homepage.tonline.de/ERPref.html> [accessed May 2, 2005].

\section{AUTHOR BIOGRAPHIES}

ROBERTO MOSCA is Full Professor of "Industrial Plants Management" and "Economy and Business Organization" at the DIP (Department of Industrial Production \& Engineering, former Institute of Technology and Mechanical Plants), University of Genoa. He has worked in the simulation sector since 1969 using discrete and stochastic industrial simulators for off-line and on-line applications. His research work focuses on the evaluation of simulation languages and new modeling techniques and his research team is developing new AI applications for industrial plant management. Currently he is directly involved as coordinator in the construction of a new campus in Savona, focused on industrial engineering. He was Director of ITIM (Institute for Technology, Industrial Engineering and Manufacturing, Istituto di Tecnologie ed Impianti Meccanici), DIP (Department of Industrial Production, Dipartimento di Ingegneria della Produzione) and DIPEM (Department of Industrial Production, Engineering and Mathematical Modelling), Dipartimento di Ingegneria della Produzione e Modelli Matematici) in Genoa University. Currently he is Director of DIPTEM (Department of Industrial Production, Technology, Engineering and Modelling), Dipartimento di Ingegneria della Produzione, Termotecnica e Modelli Matematici) in University of Genoa.
ROBERTO REVETRIA earned his degree in mechanical engineering at the University of Genoa and he completed his master thesis in Genoa Mass Transportation Company developing an automatic system integrating ANN (Artificial Neural Networks) and simulation with the ERP (Enterprise Resource Planning) for supporting purchasing activities. He had consulting experience in modeling applied to environmental management for the new Bosch plant facility TDI Common Rail Technology in construction near Bari. During his service in the Navy as officer, he was involved in the development of WSS\&S (Weapon System Simulation \& Service) Project. He completed his PhD in Mechanical Engineering in 2001. He is currently involved, as a researcher, in the DIPTEM of Genoa University, working on advanced modeling projects applied to ERP integration and maintenance planning applied to industrial case studies (Contracting \& Engineering and Retail companies). He is active in developing projects involving simulation with special attention to HLA (High Level Architecture). In 2004 he qualified for an Associate Professor Position based on a selection procedure from Parma University.

LUCIA CASSETTARI earned her degree in management engineering in 2004 at the University of Genoa . Currently she works in the Department of Production Engineering, and Mathematical Modelling (DIPTEM) at the University of Genoa as a researcher, in the field of simulator-based applications for industrial plants; particular attention is focused on the application of DOE and Optimization techniques to industrial plant problems using Simulation. She is also active in research projects involving BPR (Business Process Reengineering), Activity Based Management and Cost Control. She is senior lecturer in the Industrial Plant Management and Business Strategy degree courses.

GIANLUCA MAGRO graduated in Mechanical Engineering from Genoa University; he had experience in BPR in Mechanical Manufacturing companies. Actually he is cooperating with University of Genoa's DIPTEM regarding advanced simulation for manufacturing for supporting complex decision support systems. 